PROCEEDINGS OF THE

AMERICAN MATHEMATICAL SOCIETY

Volume 137, Number 9, September 2009, Pages 2879-2887

S 0002-9939(09)09845-1

Article electronically published on March 27, 2009

\title{
ON SUMS INVOLVING COEFFICIENTS OF AUTOMORPHIC $L$-FUNCTIONS
}

\author{
GUANGSHI LÜ \\ (Communicated by Wen-Ching Winnie Li)
}

\begin{abstract}
Let $L(s, \pi)$ be the automorphic $L$-function associated to an automorphic irreducible cuspidal representation $\pi$ of $\mathrm{GL}_{m}$ over $\mathbb{Q}$, and let $a_{\pi}(n)$ be the $n$th coefficient in its Dirichlet series expansion. In this paper we prove that if at every finite place $p, \pi_{p}$ is unramified, then for any $\varepsilon>0$,
\end{abstract}

$$
A_{\pi}(x)=\sum_{n \leq x} a_{\pi}(n) \ll_{\varepsilon, \pi}\left\{\begin{array}{ccc}
x^{\frac{71}{192}+\varepsilon} & \text { if } & m=2, \\
x^{\frac{m^{2}-m}{m^{2}+1}+\varepsilon} & \text { if } & m \geq 3 .
\end{array}\right.
$$

\section{INTRODUCTION AND MAIN RESULTS}

Let $a(n)$ be an arithmetic function. It is an important problem in number theory to establish the asymptotic formula for the summatory function

$$
A(x)=\sum_{n \leq x} a(n) .
$$

The asymptotic behavior of $A(x)$ is often closely linked with the analytic properties of the Dirichlet series

$$
\mathcal{A}(s)=\sum_{n=1}^{\infty} a(n) n^{-s}
$$

The Langlands program predicts that the most general $L$-functions arise from automorphic representations of $\mathrm{GL}_{n}$ over a number field and that such $L$-functions can be decomposed into products of primitive automorphic $L$-functions arising from irreducible cuspidal representations of $\mathrm{GL}_{m}$ over $\mathbb{Q}$. Therefore in this paper we focus our attention on primitive automorphic $L$-functions of $\mathrm{GL}_{m}$ over $\mathbb{Q}$.

To be precise, let us recall some basic facts about primitive automorphic $L$ functions of $\mathrm{GL}_{m}$ over $\mathbb{Q}$ (see Godement and Jacquet [4, Jacquet and Shalika [8], or Rudnick and Sarnak [1]). Let $\pi$ be an automorphic irreducible cuspidal

Received by the editors December 1, 2008.

2000 Mathematics Subject Classification. Primary 11F30, 11F11, 11F66.

Key words and phrases. Automorphic $L$-function, symmetric square $L$-function, Ramanujan conjecture.

This work was supported by the National Natural Science Foundation of China (Grant No. 10701048).

(C)2009 American Mathematical Society Reverts to public domain 28 years from publication 
representation of $\mathrm{GL}_{m}$ over $\mathbb{Q}$ with unitary central character. Then $\pi$ is a restricted tensor product:

$$
\pi=\otimes_{p} \pi_{p} .
$$

To $\pi$ one associates an Euler product

$$
L(s, \pi)=\prod_{p} L\left(s, \pi_{p}\right)
$$

given by a product of local factors. Outside of a finite set of primes, $\pi_{p}$ is unramified. To every finite place $p$ where $\pi_{p}$ is unramified we associate a semisimple conjugacy class

$$
A_{\pi}(p)=\left(\begin{array}{ccc}
\alpha_{\pi, p}(1), & & \\
& \ddots & \\
& & \alpha_{\pi, p}(m)
\end{array}\right),
$$

and we define the local $L$-function for the finite place $p$ as

$$
L\left(s, \pi_{p}\right)=\operatorname{det}\left(I-p^{-s} A_{\pi}(p)\right)^{-1}=\prod_{j=1}^{m}\left(1-\alpha_{\pi, p}(j) p^{-s}\right)^{-1} .
$$

It is possible to write the local factors at ramified primes $p$ in the form of (1.2) with the convention that some of the $\alpha_{\pi, p}(j)$ 's may be zero. In fact, the local factors at the ramified primes can best be described by the Langlands parameters of $\pi_{p}$.

The general Ramanujan conjectures for cuspidal automorphic representations $\pi$ of $\mathrm{GL}_{m}$ over $\mathbb{Q}$ assert that for $p$ unramified, $\left|\alpha_{\pi, p}(j)\right|=1$. For certain $\pi$, this conjecture has been proved. But in general it is still open. In this direction, Serre 12. first observed that the analytic properties of the Rankin-Selberg $L$-function, in conjunction with Landau's lemma, can lead to

$$
\left|\alpha_{\pi, p}(j)\right| \leq p^{1 / 2-1 /\left(m^{2}+1\right)} .
$$

For $m=2$, this has been refined in 9 to

$$
\left|\alpha_{\pi, p}(j)\right| \leq p^{\frac{7}{64}} .
$$

The product (1.1) over primes gives a Dirichlet series representation: for $\operatorname{Re} s>1$,

$$
L(s, \pi)=\sum_{n=1}^{\infty} \frac{a_{\pi}(n)}{n^{s}} .
$$

The aim of this paper is to study the summatory function for the coefficients $a_{\pi}(n)$ of automorphic $L$-functions attached to automorphic irreducible cuspidal representations of $\mathrm{GL}_{m}$ over $\mathbb{Q}$, i.e.

$$
A_{\pi}(x)=\sum_{n \leq x} a_{\pi}(n) .
$$

Our main result is the following.

Theorem 1.1. Let $L(s, \pi)$ be the automorphic $L$-function associated to an automorphic irreducible cuspidal representation $\pi$ of $\mathrm{GL}_{m}$ over $\mathbb{Q}$, and let $a_{\pi}(n)$ be its $n$th coefficient in (1.5). If at every finite place $p, \pi_{p}$ is unramified, then we have that for any $\varepsilon>0$,

$$
A_{\pi}(x)=\sum_{n \leq x} a_{\pi}(n) \ll_{\varepsilon, \pi}\left\{\begin{array}{ccc}
x^{\frac{71}{192}+\varepsilon} & \text { if } & m=2, \\
x^{\frac{m^{2}-m}{m^{2}+1}+\varepsilon} & \text { if } & m \geq 3,
\end{array}\right.
$$


where throughout this paper the notation $\ll_{\varepsilon, \pi}$ means that the implied constant depends on $\varepsilon$ and $\pi$.

Our Theorem 1.1, for which the Ramanujan-Petersson conjecture is not known to hold, can be compared with the results of Iwaniec and Friedlander [3]: if the Ramanujan-Petersson conjecture is assumed, then the coefficients $a(n)$ of a general $L$-function of degree $m$ with a functional equation and suitable analytic properties satisfy

$$
\sum_{n \leq x} a(n)=\text { main term }+O_{L}\left(x^{\frac{m-1}{m+1}+\varepsilon}\right) .
$$

Our result can also be compared with one result of Miller [10], which states that for any $\varepsilon>0$ and any real number $\alpha$,

$$
\sum_{n \leq x} a(m, n) e(n \alpha) \ll_{\varepsilon, m, \Phi} x^{\frac{3}{4}+\varepsilon},
$$

where $a(m, n)$ are the Fourier coefficients of a cusp form $\Phi$ for $G L(3, \mathbb{Z}) \backslash G L(3, \mathbb{R})$.

As an application of our Theorem 1.1, we shall consider the sum

$$
\sum_{n \leq x} t\left(n^{2}\right)
$$

where $t(n)$ is the $n$th normalized Fourier coefficient of a Hecke-Maass cusp form $\varphi$ corresponding to the eigenvalue $l=\kappa^{2}+\frac{1}{4}$ with respect to the full modular group $S L(2, \mathbb{Z})$, which coincides with the eigenvalue of the $n$th Hecke operator $T_{n}$.

Corollary 1.2. Let $t(n)$ be the $n$th normalized Fourier coefficient of a Hecke-Maass cusp form $\varphi$ with respect to the full modular group $S L(2, \mathbb{Z})$. Then for any $\varepsilon>0$, we have

$$
S(x)=\sum_{n \leq x} t\left(n^{2}\right) \ll_{\varepsilon, \varphi} x^{\frac{3}{5}+\varepsilon},
$$

where throughout this paper the notation $\ll_{\varepsilon, \varphi}$ means that the implied constant depends on $\varepsilon$ and the Maass cusp form $\varphi$.

Our result improves a previous result given by Ivić [6]:

$$
S(x) \ll_{\varphi} x \exp \left(-A(\log x)^{\frac{3}{5}}(\log \log x)^{-\frac{1}{5}}\right),
$$

where $A>0$ is a suitable constant.

\section{ThreE LEMMAS}

To prove Theorem 1.1, we need the following three lemmas.

Lemma 2.1. Let $L(f, s)$ be a Dirichlet series with Euler product of degree $m \geq 1$, which is defined by

$$
L(f, s)=\sum_{n=1}^{\infty} \lambda_{f}(n) n^{-s}=\prod_{p<\infty} \prod_{j=1}^{m}\left(1-\frac{\alpha_{f}(p, j)}{p^{s}}\right)^{-1},
$$

where $\alpha_{f}(p, j), j=1, \cdots, m$, are the local parameters of $L(f, s)$ at prime $p$. This series and Euler product are absolutely convergent for $\operatorname{Res}>1$. Let the gamma 
factor be given by

$$
L_{\infty}(f, s)=\prod_{j=1}^{m} \pi^{-\frac{s+\mu_{f}(j)}{2}} \Gamma\left(\frac{s+\mu_{f}(j)}{2}\right),
$$

where $\mu_{f}(j), j=1, \cdots, m$, are the local parameters of $L(f, s)$ at $\infty$. We also define the completed L-function $\Lambda(f, s)$ by

$$
\Lambda(f, s)=q(f)^{\frac{s}{2}} L_{\infty}(f, s) L(f, s)
$$

where $q(f)$ is the conductor of $L(f, s)$. We assume that $\Lambda(f, s)$ admits an analytic continuation to the whole complex plane $\mathbb{C}$ and is an entire function. Assume that it also satisfies a functional equation

$$
\Lambda(f, s)=\epsilon_{f} \Lambda(\tilde{f}, 1-s)
$$

where $\epsilon_{f}$ is the root number with $\left|\epsilon_{f}\right|=1$ and $\tilde{f}$ is the dual of $f$ such that $\lambda_{\tilde{f}}(n)=$ $\overline{\lambda_{f}(n)}, \mu_{\tilde{f}}(j)=\overline{\mu_{f}(j)}$, and $q(\tilde{f})=q(f)$.

Then for every $\eta \geq 0$ we have

$$
\sum_{n \leq x} \lambda_{f}(n) \ll_{f} x^{\frac{1}{2}-\frac{1}{2 m}+\left(\frac{m}{2}-\frac{1}{2}\right) \eta}+\sum_{x<n \leq x+x^{1-\frac{1}{m}-\eta}}\left|\lambda_{f}(n)\right| .
$$

Proof. This is a special case of Theorem 4.1 in Chandrasekharan and Narasimhan 2] with

$$
\delta=1, \quad A=\frac{m}{2}, \quad \beta=1, \quad u=\frac{1}{2}-\frac{1}{2 m} \quad \text { and } \quad q=-\infty .
$$

We reformulate it in the language used in Chapter 5 of Iwaniec and Kowalski 7 .

Lemma 2.2. With the same notation as in Lemma 2.1, we assume that the Dirichlet series $L(f, s)$ with Euler product of degree $m \geq 1$ has non-negative coefficients, i.e. $\lambda_{f}(n) \geq 0$, and converges for $\operatorname{Res}$ sufficiently large. Suppose further that $L(f, s)$ has a meromorphic continuation to $\mathbb{C}$ with, at most, poles of finite order at $s=0,1$. Assume also that $L(f, s)$ is of finite order and satisfies a functional equation

$$
\Lambda(f, s)=\epsilon_{f} \Lambda(f, 1-s) .
$$

Then we have that for any $\varepsilon>0$,

$$
\sum_{n \leq x} \lambda_{f}(n)=P(\log x) x+O_{\varepsilon, f}\left(x^{\frac{m-1}{m+1}+\varepsilon}\right),
$$

where $P$ is a polynomial depending only on $L$, whose degree equals the order of the pole of $L(f, s)$ at $s=1$.

Proof. This is a refined version of Landau's lemma; see Barthel and Ramakrishnan [1].

Lemma 2.3. Let $b(1), b(2), \ldots$ be a sequence of complex numbers. Define the sequence $a(0)=1, a(1), a(2), \ldots$ by means of the formal identity

$$
\exp \left(\sum_{k=1}^{\infty} \frac{b(k)}{k} x^{k}\right)=\sum_{n=0}^{\infty} a(n) x^{n} .
$$


For $j=1$ or 2 , define the sequence $A_{j}(0)=1, A_{j}(1), A_{j}(2), \ldots$ by means of the formal identity

$$
\exp \left(\sum_{k=1}^{\infty} \frac{|b(k)|^{j}}{k} x^{k}\right)=\sum_{n=0}^{\infty} A_{j}(n) x^{n} .
$$

Then $A_{j}(n) \geq|a(n)|^{j}$.

Proof. See Lemma 3.1 in Soundararajan [13.

\section{Proof of Theorem 1.1}

Associated with $\pi$, an automorphic representation of $\mathrm{GL}_{m}$ over $\mathbb{Q}$, there is also an Archimedean $L$-factor defined as

$$
L\left(s, \pi_{\infty}\right)=\prod_{j=1}^{m} \pi^{-\frac{s+\mu_{\pi}(j)}{2}} \Gamma\left(\frac{s+\mu_{\pi}(j)}{2}\right),
$$

where $\mu_{\pi}(j), j=1,2,3, \cdots, m$, are local parameters at $\infty$. In connection with (1.1), the completed $L$-function associated to $\pi$ is defined by

$$
\Lambda(s, \pi)=L\left(s, \pi_{\infty}\right) L(s, \pi) .
$$

This completed $L$-function has analytic continuation, is entire everywhere (note that in our case $m \geq 2$ ), and satisfies the functional equation

$$
\Lambda(s, \pi)=\epsilon_{\pi} q_{\pi}^{\frac{1}{2}-s} \Lambda(1-s, \tilde{\pi}),
$$

where $\tilde{\pi}$ is the contragredient of $\pi, \epsilon_{\pi}$ is a complex number of modulus 1 , and $q_{\pi}$ is a positive integer called the arithmetic conductor of $\pi$. For any place $p \leq \infty, \tilde{\pi}_{p}$ is equivalent to the complex conjugate $\overline{\pi_{p}}$, and we have

$$
\left\{\alpha_{\tilde{\pi}, p}(j)\right\}=\left\{\overline{\alpha_{\pi, p}(j)}\right\}, \quad\left\{\mu_{\tilde{\pi}}(j)\right\}=\left\{\overline{\mu_{\pi}(j)}\right\} .
$$

Therefore, from Lemma 2.1 and (3.1), we have

$$
A_{\pi}(x)=\sum_{n \leq x} a_{\pi}(n) \ll_{\pi} x^{\frac{1}{2}-\frac{1}{2 m}+\left(\frac{m}{2}-\frac{1}{2}\right) \eta}+\sum_{x<n \leq x+x^{1-\frac{1}{m}-\eta}}\left|a_{\pi}(n)\right|,
$$

for every $\eta \geq 0$.

For $m=2$, from (1.4) we have

$$
\left|a_{\pi}(n)\right| \leq \tau(n) n^{\frac{7}{64}},
$$

where $\tau(n)$ is the divisor function. From (3.2) with $m=2$, we have

$$
A_{\pi}(x)=\sum_{n \leq x} a_{\pi}(n) \ll_{\pi} x^{\frac{1}{4}+\frac{1}{2} \eta}+\sum_{x<n \leq x+x^{\frac{1}{2}-\eta}}\left|a_{\pi}(n)\right| .
$$

From (3.3), we obtain

$$
A_{\pi}(x) \ll_{\pi} x^{\frac{1}{4}+\frac{1}{2} \eta}+x^{\frac{39}{64}-\eta+\varepsilon} .
$$

On taking $\eta=\frac{23}{96}$, we have

$$
A_{\pi}(x) \ll_{\pi} x^{\frac{71}{192}+\varepsilon}
$$


In order to give the result for $m \geq 3$, we recall some basic facts about the Rankin-Selberg $L$-function $L(s, \pi \times \tilde{\pi})$ associated to $\pi$ and its contragredient $\tilde{\pi}$. It is defined as a product of local factors:

$$
L(s, \pi \times \tilde{\pi})=\prod_{p} L\left(s, \pi_{p} \times \tilde{\pi}_{p}\right) .
$$

For unramified primes $p$, the local factor is given by

$$
L\left(s, \pi_{p} \times \tilde{\pi}_{p}\right)=\prod_{j=1}^{m} \prod_{k=1}^{m}\left(1-\alpha_{\pi, p}(j) \overline{\alpha_{\pi, p}(k)} p^{-s}\right)^{-1} .
$$

It can be defined similarly at primes $p$ where $\pi_{p}$ is ramified. By (1.3), the product $\prod_{p} L\left(s, \pi_{p} \times \tilde{\pi}_{p}\right)$ converges absolutely on $\operatorname{Re} s>2-\frac{2}{m^{2}+1}$ (in fact on Res $>1$; see e.g. Jacquet and Shalika [8] or Rudnick and Sarnak [1] ). We write this product as a Dirichlet series:

$$
L(s, \pi \times \tilde{\pi})=\prod_{p} \sum_{k=0}^{\infty} \frac{a_{\pi \times \tilde{\pi}}\left(p^{k}\right)}{p^{k s}}=\sum_{n=1}^{\infty} \frac{a_{\pi \times \tilde{\pi}}(n)}{n^{s}} .
$$

The completed Rankin-Selberg $L$-function is defined by

$$
\Lambda(s, \pi \times \tilde{\pi})=L\left(s, \pi_{\infty} \times \tilde{\pi}_{\infty}\right) L(s, \pi \times \tilde{\pi})
$$

with

$$
L\left(s, \pi_{\infty} \times \tilde{\pi}_{\infty}\right)=\prod_{j=1}^{m^{2}} \pi^{-\frac{s+\mu_{\pi \times \tilde{\pi}(j)}}{2}} \Gamma\left(\frac{s+\mu_{\pi \times \tilde{\pi}}(j)}{2}\right) .
$$

When $\pi_{\infty}$ is unramified,

$$
\left\{\mu_{\pi \times \tilde{\pi}}(j)\right\}_{1 \leq j \leq m^{2}}=\left\{\mu_{\pi}(j)+\overline{\mu_{\pi}(k)}\right\}_{1 \leq j \leq m, 1 \leq k \leq m} .
$$

It is known that $a_{\pi \times \tilde{\pi}}(n) \geq 0$ and $L(s, \pi \times \tilde{\pi})$ has a simple pole at $s=1$. The completed Rankin-Selberg $L$-function $\Lambda(s, \pi \times \tilde{\pi})$ has a meromorphic continuation to the entire complex plane and satisfies a functional equation

$$
\Lambda(s, \pi \times \tilde{\pi})=\epsilon_{\pi \times \tilde{\pi}} q_{\pi \times \tilde{\pi}}^{\frac{1}{2}-s} \Lambda(1-s, \pi \times \tilde{\pi}),
$$

where $\left|\epsilon_{\pi \times \tilde{\pi}}\right|=1$ and $q_{\pi \times \tilde{\pi}}>0$.

Therefore by applying Lemma 2.2 to $L(s, \pi \times \tilde{\pi})$ with degree $m^{2}$, we have

$$
\sum_{n \leq x} a_{\pi \times \tilde{\pi}}(n)=c_{\pi} x+O_{\varepsilon, \pi}\left(x^{\frac{m^{2}-1}{m^{2}+1}+\varepsilon}\right),
$$

where $c_{\pi}$ is a positive constant.

From (3.10), we find that for any $\eta \geq 0$,

$$
\sum_{x<n \leq x+x^{1-\frac{1}{m}-\eta}} a_{\pi \times \tilde{\pi}}(n) \ll_{\varepsilon, \pi} x^{\frac{m^{2}-1}{m^{2}+1}+\varepsilon} .
$$

From (3.7), (3.8) and (3.9), we have that for $\operatorname{Re} s>2-\frac{2}{m^{2}+1}$,

$$
\sum_{k=0}^{\infty} \frac{a_{\pi \times \tilde{\pi}}\left(p^{k}\right)}{p^{k s}}=\exp \left(\sum_{v=1}^{\infty} \frac{\left|\lambda_{\pi}\left(p^{v}\right)\right|^{2}}{v} p^{-v s}\right),
$$


where

$$
\lambda_{\pi}\left(p^{v}\right)=\sum_{j=1}^{m} \alpha_{\pi, p}(j)^{v}
$$

From (1.1), (1.2) and (1.5), we have

$$
\sum_{k=0}^{\infty} \frac{a_{\pi}\left(p^{k}\right)}{p^{k s}}=\exp \left(\sum_{v=1}^{\infty} \frac{\lambda_{\pi}\left(p^{v}\right)}{v} p^{-v s}\right) .
$$

From (3.12), (3.13) and Lemma 2.3 with $j=2$, we have that for an unramified prime $p$,

$$
\left|a_{\pi}\left(p^{k}\right)\right|^{2} \leq a_{\pi \times \tilde{\pi}}\left(p^{k}\right)
$$

and thus in our case

$$
\left|a_{\pi}(n)\right|^{2} \leq a_{\pi \times \tilde{\pi}}(n)
$$

Therefore we have

$$
\sum_{x<n \leq x+x^{1-\frac{1}{m}-\eta}}\left|a_{\pi}(n)\right|^{2} \ll \sum_{x<n \leq x+x^{1-\frac{1}{m}-\eta}} a_{\pi \times \tilde{\pi}}(n) .
$$

Now we begin to estimate (3.2). By Cauchy's inequality, we find that the shortinterval sum in (3.2) satisfies$$
\sum_{x<n \leq x+x^{1-\frac{1}{m}-\eta}}\left|a_{\pi}(n)\right| \leq\left(\sum_{x<n \leq x+x^{1-\frac{1}{m}-\eta}}\left|a_{\pi}(n)\right|^{2}\right)\left(\sum_{x<n \leq x+x^{1-\frac{1}{m}-\eta}} 1\right)^{\frac{1}{2}}
$$

By (3.11) and (3.14), we have

$$
\sum_{x<n \leq x+x^{1-\frac{1}{m}-\eta}}\left|a_{\pi}(n)\right|^{2} \ll_{\varepsilon, \pi} x^{\frac{m^{2}-1}{m^{2}+1}+\varepsilon} .
$$

From (3.15) and (3.16), we obtain

$$
\sum_{x<n \leq x+x^{1-\frac{1}{m}-\eta}}\left|a_{\pi}(n)\right| \ll_{\varepsilon, \pi} x^{\frac{1}{2}-\frac{1}{2 m}-\frac{\eta}{2}+\frac{m^{2}-1}{2 m^{2}+2}+\varepsilon} .
$$

Inserting (3.17) into (3.2), we have

$$
A_{\pi}(x)=\sum_{n \leq x} a_{\pi}(n) \ll_{\varepsilon, \pi} x^{\frac{1}{2}-\frac{1}{2 m}+\left(\frac{m}{2}-\frac{1}{2}\right) \eta}+x^{\frac{1}{2}-\frac{1}{2 m}-\frac{\eta}{2}+\frac{m^{2}-1}{2 m^{2}+2}+\varepsilon} .
$$

On taking $\eta=\frac{m^{2}-1}{m\left(m^{2}+1\right)}$, we get

$$
A_{\pi}(x) \ll_{\varepsilon, \pi} x^{\frac{m^{2}-m}{m^{2}+1}+\varepsilon} .
$$

This completes the proof of Theorem 1.1. 


\section{Proof of Corollary 1.2}

To prove Corollary 1.2, we recall some basic facts from the books of Iwaniec and Kowalski [7, and of Goldfeld [5]. Associated to each Hecke-Maass cusp form $\varphi$ for the full modular group $S L(2, \mathbb{Z})$ there is an $L$-function $L(\varphi, s)$, which is defined, for $\operatorname{Re} s>1$, by

$$
\begin{aligned}
L(\varphi, s) & =\sum_{n=1}^{\infty} t(n) n^{-s}=\prod_{p}\left(1-t(p) p^{-s}+p^{-2 s}\right)^{-1} \\
& =\prod_{p}\left(1-\frac{\alpha_{p}}{p^{s}}\right)^{-1}\left(1-\frac{\alpha_{p}^{\prime}}{p^{s}}\right)^{-1}
\end{aligned}
$$

with $\alpha_{p}+\alpha_{p}^{\prime}=t(p)$ and $\alpha_{p} \alpha_{p}^{\prime}=1$. The symmetric square $L$-function $L\left(\operatorname{Sym}^{2} \varphi, s\right)$ is defined, for $\operatorname{Re} s>1$, by

$$
\begin{aligned}
L\left(\operatorname{Sym}^{2} \varphi, s\right) & =\zeta(2 s) \sum_{n=1}^{\infty} t\left(n^{2}\right) n^{-s} \\
& =\prod_{p}\left(1-\frac{\alpha_{p}^{2}}{p^{s}}\right)^{-1}\left(1-\frac{1}{p^{s}}\right)^{-1}\left(1-\frac{\alpha_{p}^{\prime 2}}{p^{s}}\right)^{-1},
\end{aligned}
$$

where $\zeta(s)$ is the Riemann zeta-function. Then we have

$$
\sum_{n=1}^{\infty} t\left(n^{2}\right) n^{-s}=\frac{L\left(\operatorname{Sym}^{2} \varphi, s\right)}{\zeta(2 s)} .
$$

This gives

$$
t\left(n^{2}\right)=\sum_{d^{2} \mid n} \mu(d) t^{(2)}\left(\frac{n}{d^{2}}\right),
$$

where $t^{(2)}(n)$ is the $n$th coefficient of the symmetric square $L$-function $L\left(\operatorname{Sym}^{2} \varphi, s\right)$ with $\operatorname{Re} s>1$.

It follows from the Gelbart-Jacquet lift that $L\left(\operatorname{Sym}^{2} \varphi, s\right)$ is an automorphic $L$ function of $\mathrm{GL}_{3}$. Then from Theorem 1.1 with $m=3$, we have

$$
\sum_{n \leq x} t^{(2)}(n) \ll x^{\frac{3}{5}+\varepsilon} .
$$

From (4.2) and (4.3), we have

$$
S(x)=\sum_{n \leq x} t\left(n^{2}\right) \ll x^{\frac{3}{5}+\varepsilon} .
$$

This completes the proof of Corollary 1.2.

\section{ACKNOWLEDGEMENTS}

I would like to thank Professor Jianya Liu and Professor K. Soundararajan for their encouragement. I am grateful to the referee of the first version of this paper and the referee of the present version for their valuable suggestions and detailed comments. 


\section{REFERENCES}

[1] L. Barthel and D. Ramakrishnan, A nonvanishing result for twists of $L$-functions of $G L(n)$, Duke Math. J., 74(1994), 681-700. MR1277950 (95d:11062)

[2] K. Chandrasekharan and R. Narasimhan, Functional equations with multiple gamma factors and the average order of arithmetical functions, Ann. of Math. (2), 76(1962), 93-136. MR0140491 (25:3911)

[3] J.B. Friedlander and H. Iwaniec, Summation formulae for coefficients of $L$-functions, Canad. J. Math., 57(2005), 494-505. MR2134400 (2006d:11095)

[4] R. Godement and H. Jacquet, Zeta functions of simple algebras, Lecture Notes in Math., 260, Springer, Berlin, 1972. MR 0342495 (49:7241)

[5] D. Goldfeld, Automorphic forms and $L$-functions for the group $G L(n, \mathbb{R})$, Cambridge Studies in Advanced Mathematics, 99, Cambridge University Press, Cambridge, 2006. MR2254662 (2008d:11046)

[6] A. Ivić, On sums of Fourier coefficients of cusp forms, Proceedings of the IV International Conference "Modern Problems of Number Theory and Its Applications": Current Problems, Part II (Tula, Russia, 2001), 92-97, Mosk. Gos. Univ. im. Lomonosova, Mekh. Mat. Fak., Moscow, 2002. MR.1985942 (2004d:11030)

[7] H. Iwaniec and E. Kowalski, Analytic number theory, Amer. Math. Soc. Colloquium Publ., 53, Amer. Math. Soc., Providence, RI, 2004. MR2061214 (2005h:11005)

[8] H. Jacquet and J.A. Shalika, On Euler products and the classification of automorphic representations. I, Amer. J. Math., 103(1981), 499-558. MR618323 (82m:10050a)

[9] H. Kim, with D. Ramakrishnan and P. Sarnak, Functoriality for the exterior square of $G L_{4}$ and the symmetric fourth of $G L_{2}$ (by Kim); with appendix, Refined estimates towards the Ramanujan and Selberg conjectures (by Kim and Sarnak), J. Amer. Math. Soc., 16(2003), 139-183. MR1937203 (2003k:11083)

[10] S.D. Miller, Cancellation in additively twisted sums on $G L(n)$, Amer. J. Math., 128(2006), 699-729. MR2230922 (2007k:11078)

[11] Z. Rudnick and P. Sarnak, Zeros of principal $L$-functions and random matrix theory, Duke Math. J., 81(1996), 269-322. MR1395406 (97f:11074)

[12] J-P. Serre, Letters to J.-M. Deshouillers (1981).

[13] K. Soundararajan, Weak subconvexity for central values of $L$-functions, arXiv.0809.1635vl. Submitted to Ann. of Math. (2).

Department of Mathematics, Shandong University, Jinan, Shandong 250100, People's Republic of China

E-mail address: gslv@sdu.edu.cn 